\title{
Developing a MOOC to Foster Information Literacy (IL) by Means of a Conjecture Map
}

\section{LTEC 2019}

Prof. Dr. Sabine Seufert

Dr. Josef Guggemos

Luca Moser

17.07.2019 
Overview

(1) $\mathrm{i}-\mathrm{MOOC}$

2 Information Literacy (IL) Framework

2 i-MOOC bases on the 7i-model of information literacy (IL)

3 Method

The conjecture map as a part of our educational design research cycle

4 i-MOOC Conjecture Map

Explication of the underlying assumptions

$5 \quad$ First findings

Collaboration | Efficiency | ...

$6 \quad$ Further Work and Discussion

6 MOOC-Design | Institutional requirements | ... 

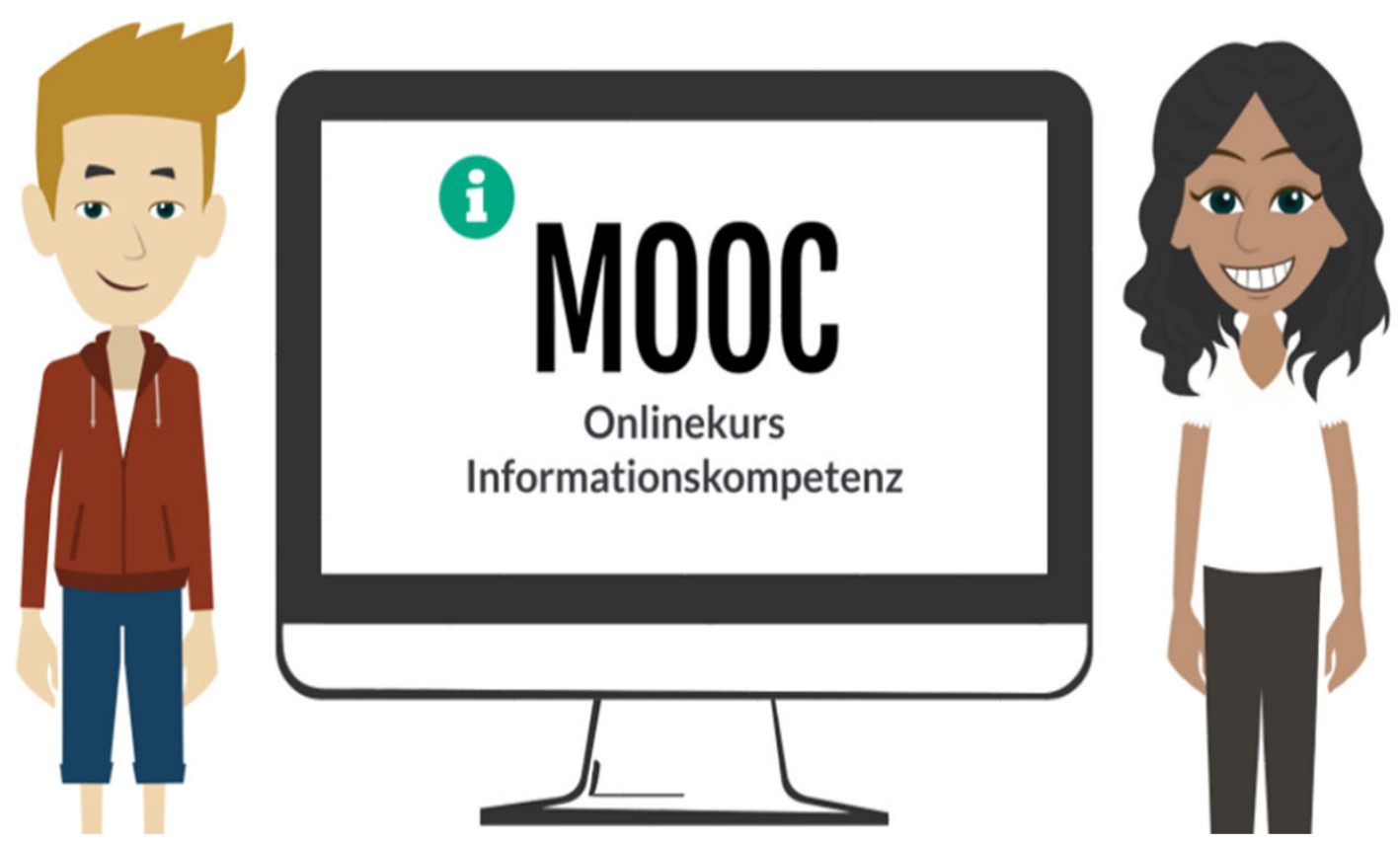

950 Enrolled

620 Active

200 Completed

21 Schools

29 Teachers

54 Classes

Massive Open Online Course to foster Information Literacy

for: High School Students in Switzerland (Sekundarstufe II)

Foster Information Literacy Science Communication 
Information Literacy (IL) Framework | 7i-Model (Seufert et al., 2015)

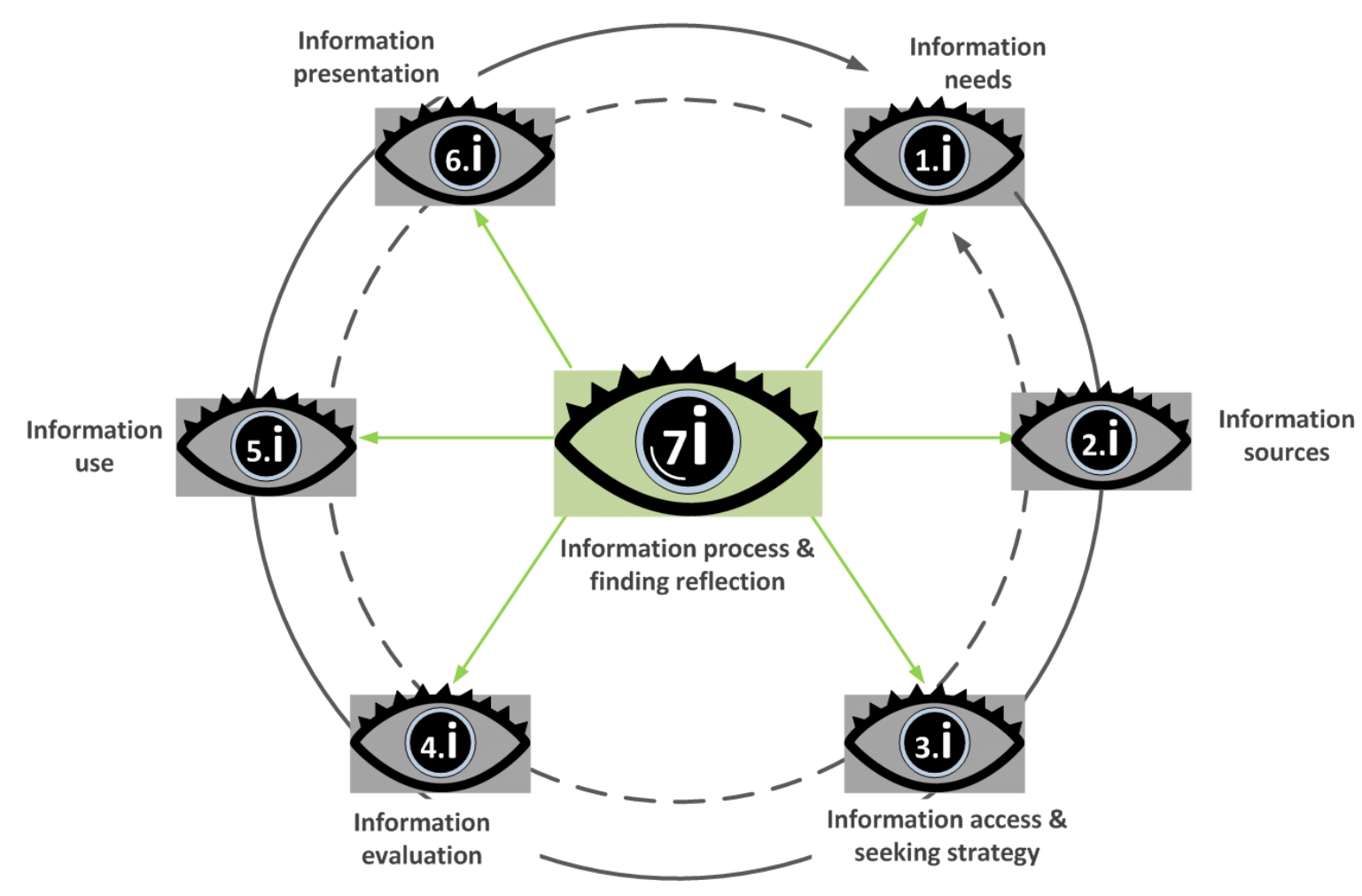

\begin{tabular}{|c|c|c|}
\hline \multicolumn{3}{|c|}{ Course Structure and Levels } \\
\hline Module & Content & Level \\
\hline M1 & $\begin{array}{l}\text { - Introduction } \\
\text { - } \quad \text { Pretest }\end{array}$ & Info Tourist \\
\hline M2 & $\begin{array}{l}\text { - I-needs } \\
\text { - I-sources }\end{array}$ & Info Mapper \\
\hline M3 & $\begin{array}{ll}\text { - } & \text { I-access } \\
\text { - } & \text { I-evaluation }\end{array}$ & Info User \\
\hline M4 & $\begin{array}{ll}\text { - } & \text { I-use } \\
\text { - } & \text { I-presentation }\end{array}$ & Info Guide \\
\hline M5 & - Internet Rally & Info Leader \\
\hline M6 & - Posttest & Info Master \\
\hline
\end{tabular}


Method | Educational Design Research Cycle (Wozniak 2015; Mc Kenney \& Reeves, 2012)

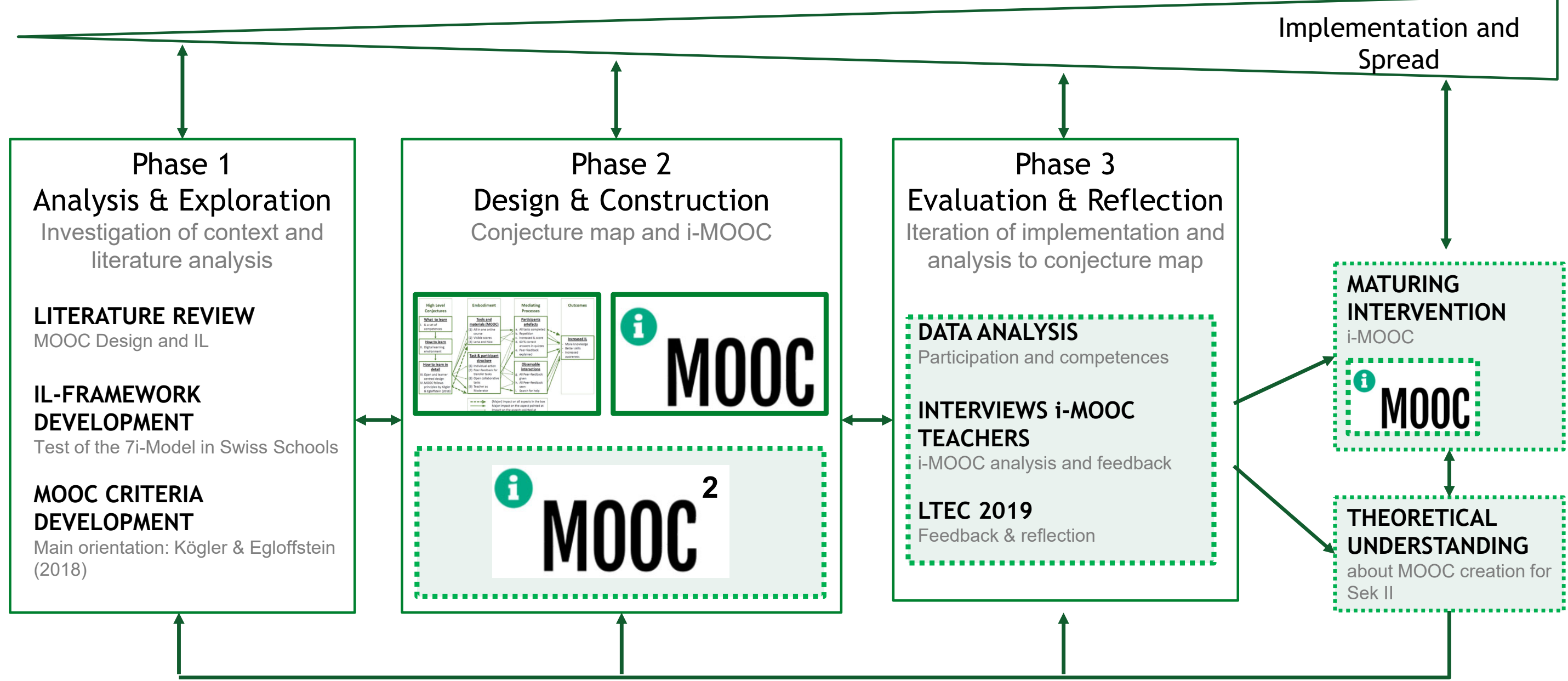




\section{Method | Conjecture Map}
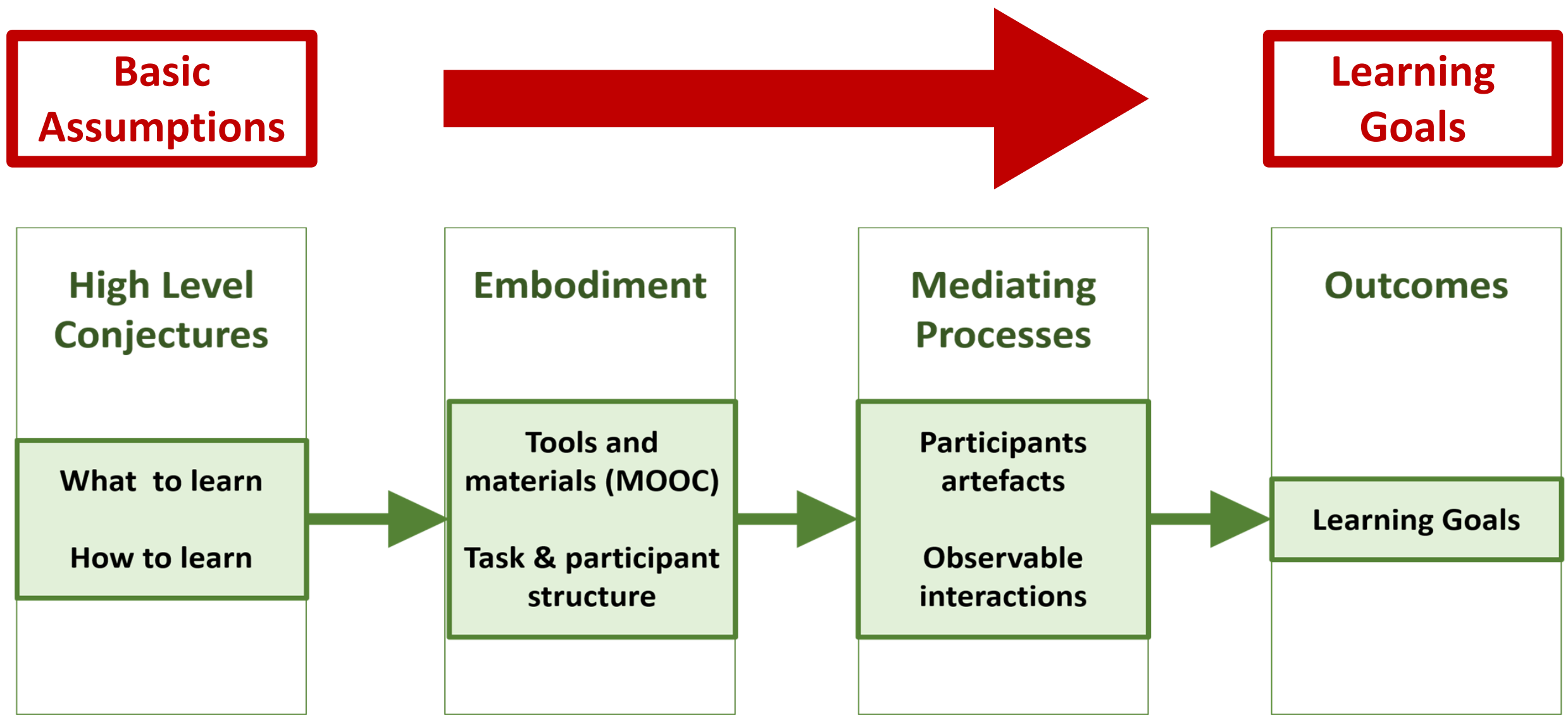
i-MOOC Conjecture Map (based on: Sandoval, 2014 / Wozniak 2015)

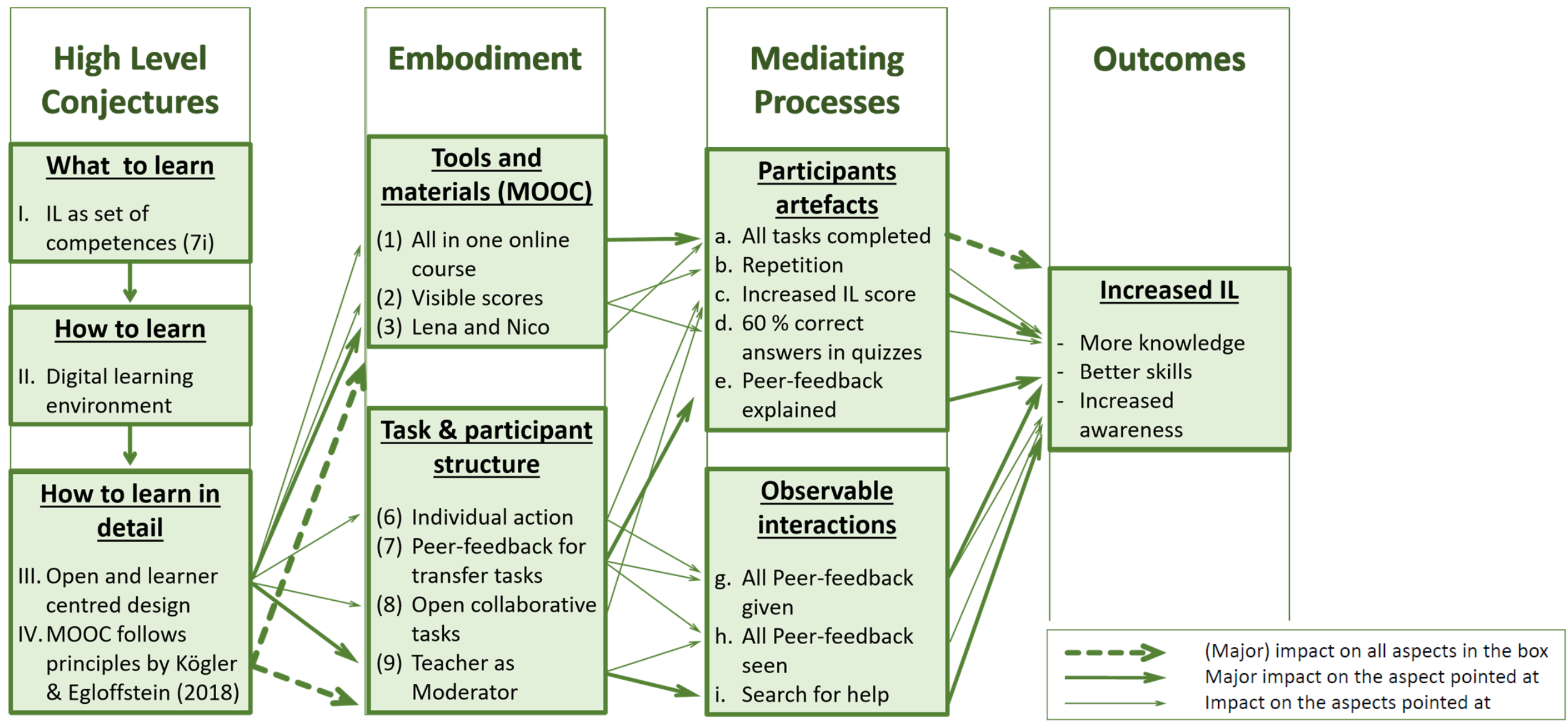


First Findings | Interviews

DESIGN PRINCIPLES

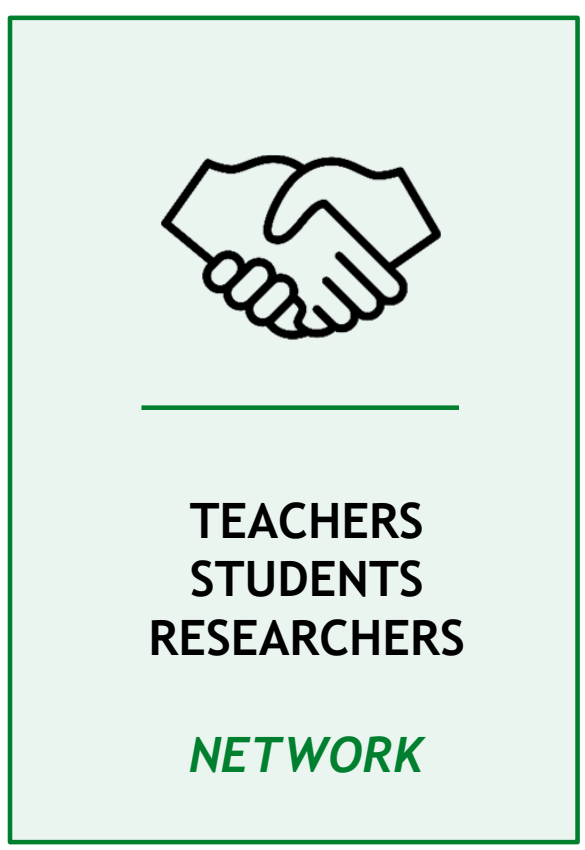

INSTITUTIONAL

LEVERS

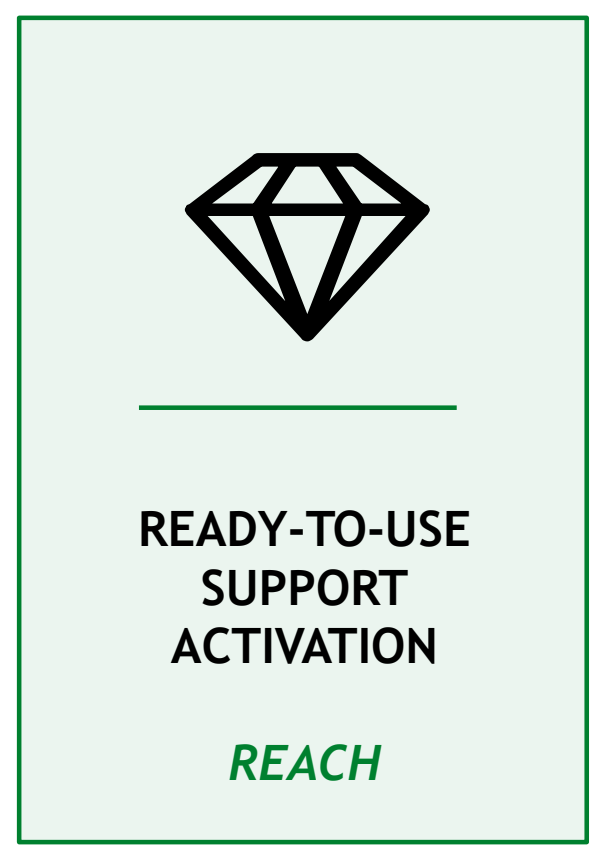

POTENTIAL

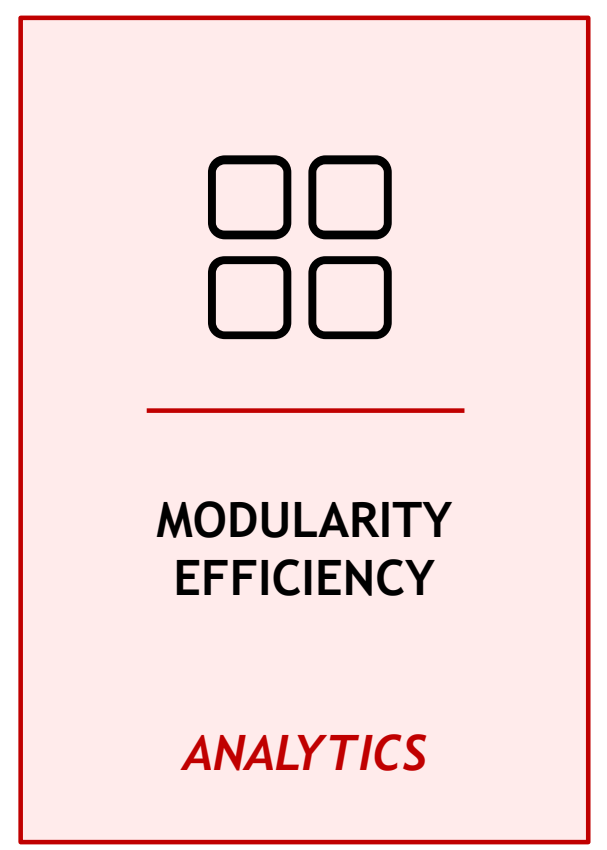

CHALLENGES

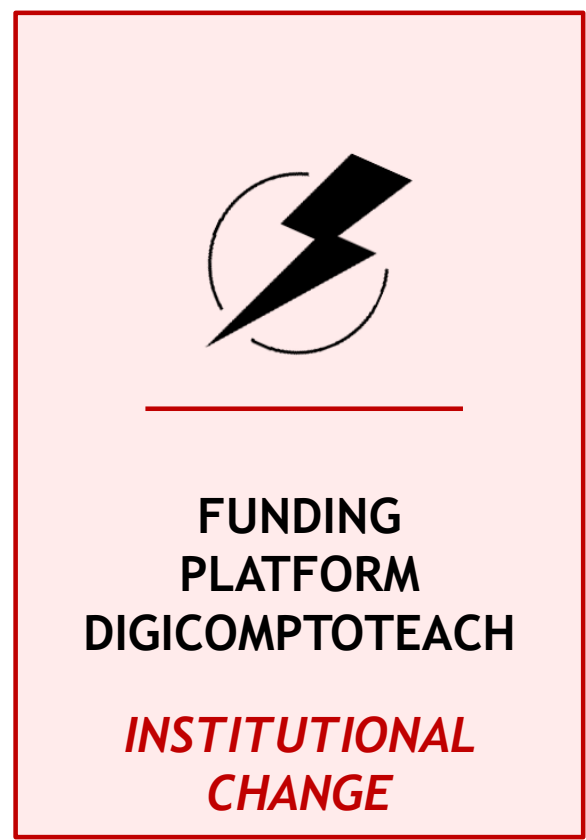




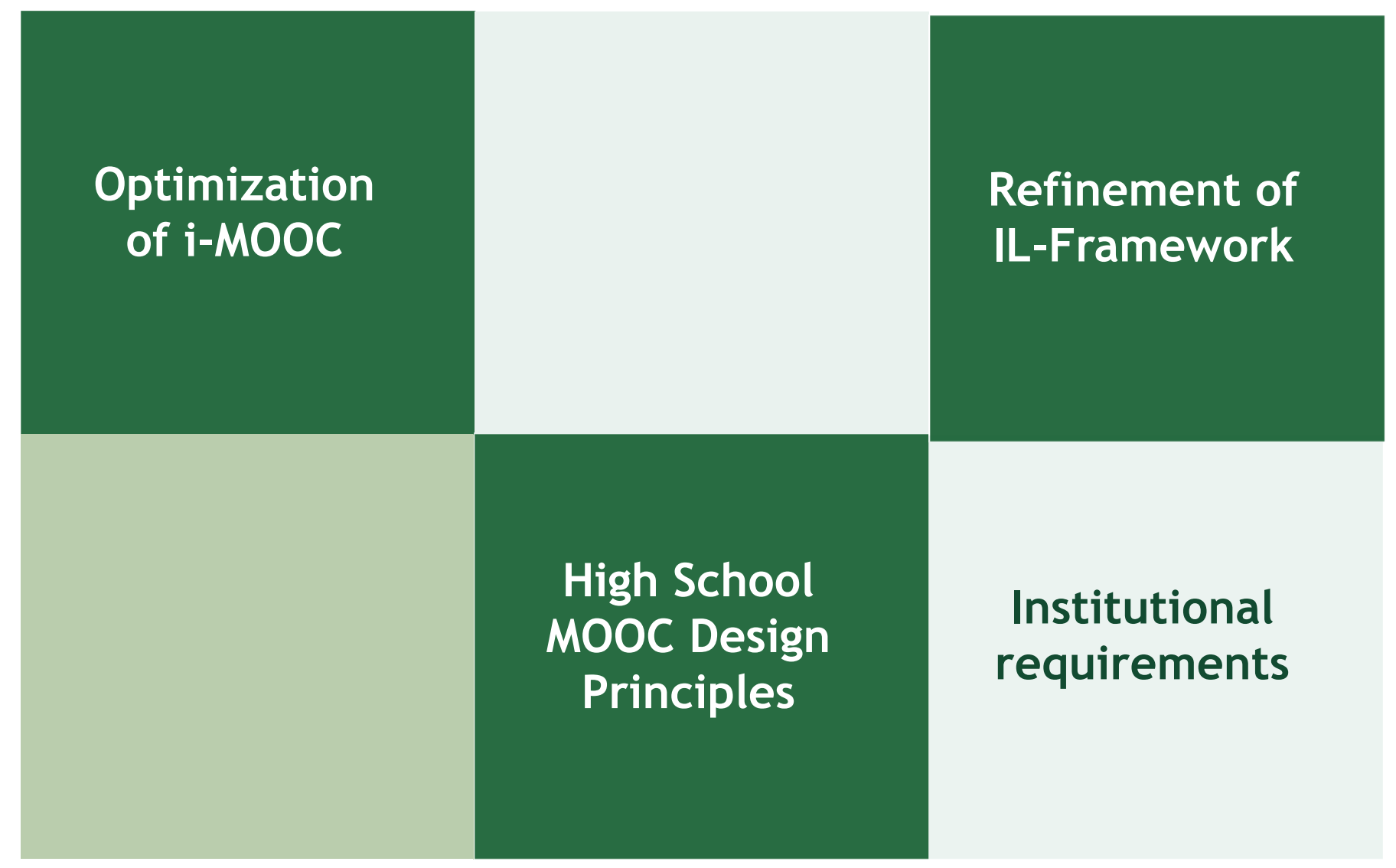




\section{Literature}

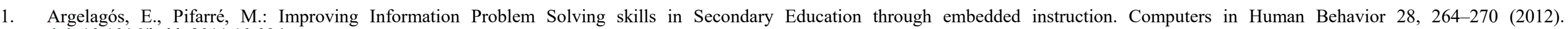
doi: $10.1016 /$ j.chb.2011.10.024

2. Balceris, M.: Medien- und Informationskompetenz: Modellierung und Messung von Informationskompetenz bei Schülern. Dissertation, Freie Universität Berlin (2011)

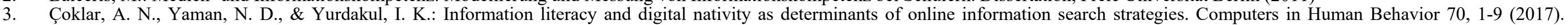
doi: 10.1016/j.chb.2016.12.050

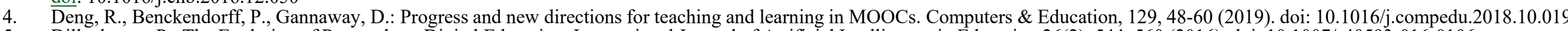

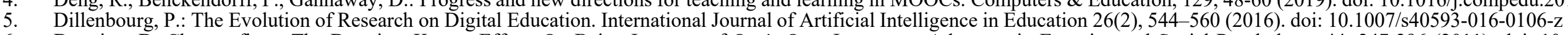

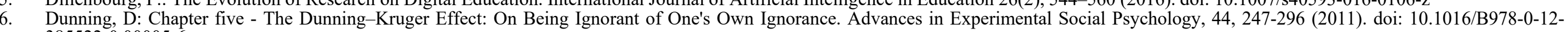
385522-0.00005-6

7. Euler, D.: Wirkungs- vs. Gestaltungsforschung - eine feindliche Koexistenz? Zeitschrift für Berufs- und Wirtschaftspädagogik 107(4), 520-542 (2011)

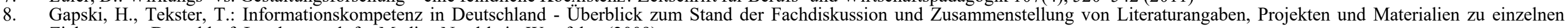
Zielgruppen. Düsseldorf: Landesanstalt für Medien Nordrhein-Westfalen (2009).

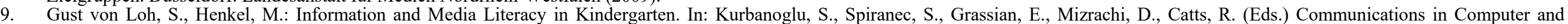
Information Science: vol. 492, pp. 253-262. Berlin: Springer International Publishing (2014). doi: 10.1007/978-3-319-14136-7 27

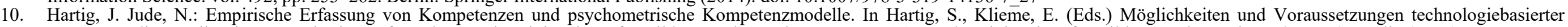
Kompetenzdiagnostik. Eine Expertise im Auftrag des Bundesministeriums für Bildung und Forschung, pp. 17-36. Bundesministerium für Bildung und Forschung (BMBF): Berlin (2007)

11. Hattie, J., Timperley, H.: The Power of Feedback. Review of Educational Research, 77(1), 81-112 (2007). doi: 10.3102/003465430298487

12. HSG (University of St. Gallen) Homepage. https://www.unisg.ch/ueber-uns/portraet/rankingsundakkreditierungen/rankingresultate, last accessed 2019/01/03

13. Jones-Kavalier, B., and Flannigan, S.: Connecting the digital dots: Literacy of the 21st century. Educause Quarterly (2), 8-10 (2006)

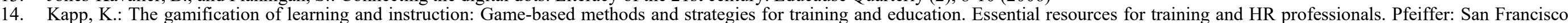
(2012)

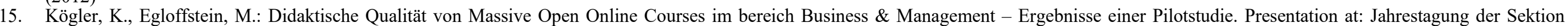
Berufs- und Wirtschaftspädagogik 2018, Frankfurt (2018)

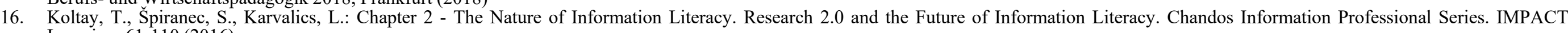
Learning, 61-110(2016)

17. Lanning, S., Mallek, J.: Factors Influencing Information Literacy Competency of College Students. The Journal of Academic Librarianship, 43(5), 443-450 (2017)

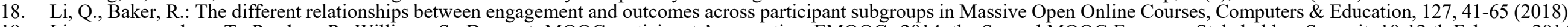

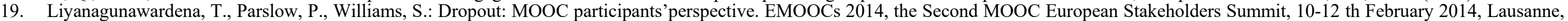
Switzerland, 95-100 (2014)

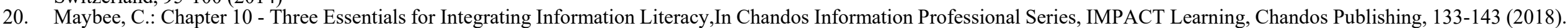




\section{Literature}

21. McKenney, S., Reeves, T.: Conducting educational design research. Routledge: Oxon (2012)

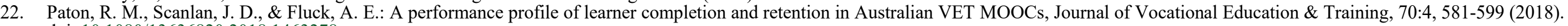
doi: $10.1080 / 13636820.2018 .1463278$

23. Prensky, M.: Digital natives, digital immigrants. MCB University Press, 9(5), 1-6 (2011). doi: 10.1108/10748120110424816

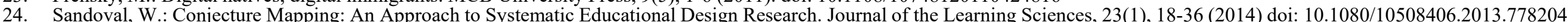

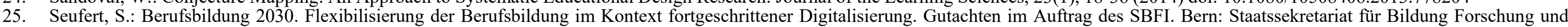
Innovation (2018)

26. Seufert, S.: Potenziale von Design Research aus der Perspektive der Innovationsforschung. Zeitschrift für Berufs- und Wirtschaftspädagogik, Beiheft 2(27), 79-96 (2014).

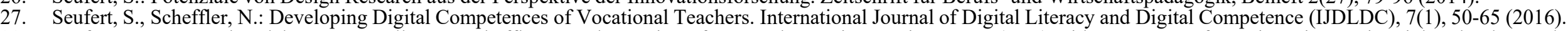

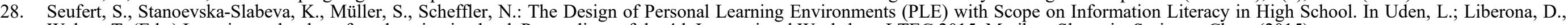
Welzer, T. (Eds.) Learning technology for education in cloud. Proceedings of the 4th International Workshop, LTEC 2015, Maribor, Slovenia, Springer: Cham (2015)

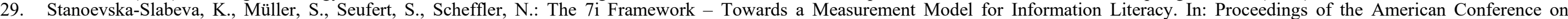
Information Systems (AMCIS), pp. 1-14, Fajardo, PR (2015)

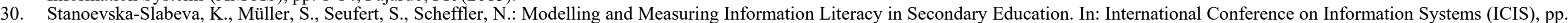
$1-20$, Fort Worth, Texas (2015)

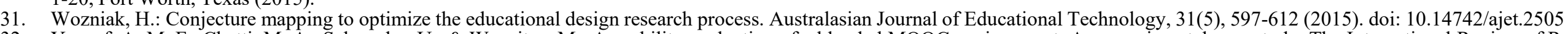

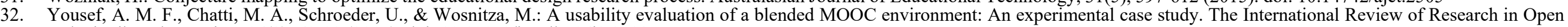
and Distributed Learning, 16(2), 69-93 (2015). doi: 10.19173/irrodl.v16i2.2032 